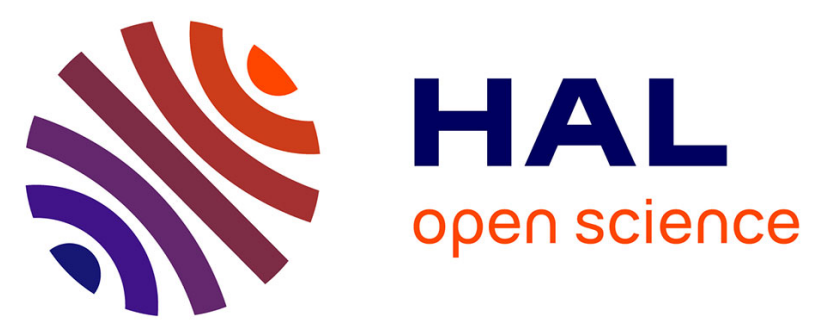

\title{
Réduction des rejets azotés des porcs par la voie alimentaire : évaluation économique et influence des changements de la Politique Agricole Commune
}

\author{
Jean-Yves Dourmad, Chantal Le Mouël, P. Rainelli
}

\section{- To cite this version:}

Jean-Yves Dourmad, Chantal Le Mouël, P. Rainelli. Réduction des rejets azotés des porcs par la voie alimentaire: évaluation économique et influence des changements de la Politique Agricole Commune. Productions Animales, 1995, 8 (2), pp.135-144. hal-00896111

HAL Id: hal-00896111

https://hal.science/hal-00896111

Submitted on 1 Jan 1995

HAL is a multi-disciplinary open access archive for the deposit and dissemination of scientific research documents, whether they are published or not. The documents may come from teaching and research institutions in France or abroad, or from public or private research centers.
L'archive ouverte pluridisciplinaire HAL, est destinée au dépôt et à la diffusion de documents scientifiques de niveau recherche, publiés ou non, émanant des établissements d'enseignement et de recherche français ou étrangers, des laboratoires publics ou privés. 
INRA Prod. Anim., $1995,8(2), 135-144$

\section{J.Y. DOURMAD, C. LE MOUËL *, P. RAINELLI *}

INRA Station de Recherches Porcines, 35590 Saint-Gilles

* INRA Station d'Economie et Sociologie Rurales, 65 rue de St-Brieuc, 35000 Rennes
Réduction des rejets azotés des porcs par la voie alimentaire : évaluation économique et influence des changements de la Politique Agricole Commune

\section{La réforme de la PAC modifie la hiérarchie des prix des matières premières de l'alimentation animale. Cela a des conséquences sur la formulation des régimes et leurs teneurs en protéines, qui conditionnent les rejets azotés.}

L'élimination des déjections animales constitue un problème crucial dans plusieurs régions européennes à forte densité de pro-

\section{Résumé}

Dans les régions de productions animales intensives, l'élimination des déjections constitue un problème crucial, en raison principalement des risques de pollution des eaux par les nitrates et de l'air par l'ammoniac. On a envisagé récemment, comme alternative ou en complément du traitement des effluents, des solutions préventives visant à réduire les rejets azotés à la source. Il s'agit principalement de mieux adapter l'apport protéique de l'aliment et d'améliorer les performances des animaux. Dans le contexte de la nouvelle politique agricole commune (PAC), nous avons tenté d'évaluer le coût de cette approche préventive. Les résultats montrent que la réforme de la PAC induit des modifications importantes dans la formulation des régimes. On constate ainsi une réduction de 1 à 1,5 point de la teneur en protéines des aliments qui s'accompagne d'une diminution de l'excrétion azotée de 0,2 à $0,4 \mathrm{~kg} /$ porc. Cependant, cette évolution est très sensible aux rapports de prix entre sources de protéines et d'énergie. La modification de la conduite de l'alimentation permet de réduire le rejet azoté jusqu'à 500 g/porc, tout en réduisant le coût « matières premières" de l'aliment de 8 à $13 \mathrm{~F} /$ porc $(3$ à $5 \%$ ). Mais cette approche induit des investissements supplémentaires au niveau de l'élevage en terme de stockage et de distribution d'aliment. L'amélioration de l'équilibre du régime en acides aminés s'accompagne d'une augmentation du coût alimentaire d'autant plus importante que l'on s'éloigne de la solution optimale. Une réduction du rejet azoté de $500 \mathrm{~g} /$ porc entraîne ainsi un coût supplémentaire de 2 à 4 F/porc. Si l'on combine l'amélioration de la stratégie d'alimentation et l'amélioration de l'équilibre protéique du régime, on peut réduire le rejet azoté d'environ 20 à $25 \%$ sans augmenter significativement le coût matières premières de l'aliment. duction, en raison principalement des risques de pollution des eaux par les nitrates et de l'air par les émanations d'ammoniac. Les techniques de traitement visant à l'épuration totale ou partielle des effluents ont été largement étudiées, mais leur développement est resté limité, en raison des coûts élevés d'investissement et de fonctionnement. Plus récemment, on a envisagé des solutions préventives visant à réduire les rejets azotés à la source (Lenis 1987, Dourmad et Henry 1994). Ainsi, la distribution d'un aliment adapté à chaque stade physiologique permet de réduire les rejets azotés de 15 à $20 \%$ par rapport à la situation actuelle. De même, l'amélioration de la qualité des protéines, grâce en particulier à la supplémentation en acides aminés industriels, laisse envisager une réduction supplémentaire de 15 à $20 \%$ des rejets azotés. L'amélioration du niveau des performances, outre son intérêt économique, conduit également à une baisse des rejets.

Cette approche préventive est incontestablement d'un grand intérêt pour l'environnement, mais elle comporte aussi un impact économique qu'il convient d'évaluer avant son application pratique. De plus, le coût des différentes solutions proposées est étroitement dépendant du prix relatif des différents ingrédients entrant dans la composition de l'ali- 
ment. Il est donc nécessaire de considérer l'évolution du prix des différentes matières premières, d'autant plus que le contexte actuel de modification de la Politique Agricole Commune (PAC) s'accompagne d'une évolution très rapide du marché. Après une présentation rapide du contexte général de la $\mathrm{PAC}$ en relation avec l'alimentation animale, nous en analyserons les effets sur la composition des régimes et les rejets azotés des porcs pour différentes hypothèses de conduite alimentaire.

\section{1 / Alimentation animale et politique agricole}

Au cours des deux dernières décennies, l'important soutien communautaire aux céréales, par le biais de prix élevés, a rendu cet ingrédient relativement cher par rapport aux autres matières servant à fabriquer les aliments composés, notamment le soja et les produits de substitution comme le corn gluten feed (CGF). Ces autres matières premières étant plus riches en protéines que les céréales, ce phénomène de substitution a entraîné une augmentation graduelle de la teneur en protéines des rations animales. Ainsi, la minimisation du coût de l'aliment a eu pour contrepartie un accroissement de la charge polluante des déjections porcines, dû à une augmentation de la quantité d'azote ingéré, alors que la quantité d'azote retenu ne changeait pas.

La réforme de la PAC adoptée en mai 1992 va sensiblement modifier la hiérarchie des prix des diverses matières premières servant ou pouvant servir à fabriquer des aliments composés. Dans ces conditions, on peut se demander si cette nouvelle hiérarchie ne va pas modifier la ration alimentaire en faveur des céréales. En d'autres termes, peut-on espérer parvenir à une réduction des rejets azotés par le simple jeu du marché sans une action volontariste des pouvoirs publics?

L'élément central de la réforme de la PAC réside dans la baisse des prix de soutien des céréales. La diminution des prix d'intervention de $35 \%$ entre le $1^{\text {er }}$ juillet 1992 et le $1^{\text {er }}$ juillet 1995 devrait en effet se traduire par une baisse du prix d'achat du blé, de l'orge, du maïs... Toutefois, leur plus grande incorporation dans l'alimentation animale dépend étroitement de l'évolution du prix des autres ingrédients susceptibles de jouer le rôle de substituts. Malheureusement cette évolution est impossible à déterminer a priori car elle dépend de l'ensemble des mesures adoptées et des nouveaux équilibres qui s'établiront.

En effet, la réforme va enclencher un ensemble complexe d'ajustements à la fois sur les marchés intérieurs et sur les marchés mondiaux. Pour les premiers, les principales modifications sont dues aux interactions entre production végétale, alimentation animale et élevage. Pour les seconds, des ajustements vont s'opérer concernant les prix mon- diaux du soja et des produits de substitution. La détermination des rapports de prix entre les principaux constituants de l'alimentation animale, une fois la réforme entrée dans les faits, ne peut donc se faire qu'à l'aide d'une modélisation globale des relations entre tous les produits, et ce au niveau mondial. MISS, Modèle International Simplifié de Simulation est particulièrement bien adapté pour cet exercice (Guyomard et Mahé 1993).

MISS a été conçu pour évaluer les conséquences des changements de politique agricole des principaux acteurs mondiaux : Union Européenne et Etats-Unis. De type walrassien, les systèmes d'offre et de demande sont guidés par les prix. Toutefois, les quotas sont représentés de manière explicite et les effets du progrès technique sont intégrés. Le calibrage des paramètres de comportement a été réalisé à partir de travaux économétriques fournissant des estimations de moyen terme (4 à 5 ans). Ainsi peut-on apprécier toute modification de politique agricole en termes de cours mondiaux et de prix intérieurs, de volumes offerts, demandés et échangés. Par simulation, on peut aussi déterminer le contexte de prix des matières premières de l'alimentation animale à l'horizon 1995/96.

Les résultats du modèle dépendent évidemment d'un ensemble d'hypothèses quant aux modalités d'application de la réforme. On en rappellera trois :

- les aides directes attribuées aux producteurs pour compenser les baisses de prix sont considérées comme des compléments de prix (hypothèse de couplage des versements compensatoires) ;

la baisse des prix à la production des céréales et des oléopréotéagineux entraîne une diminution de la tendance autonome de croissance des rendements qui sont ramenés à $1,2 \%$ par an pour les céréales et à $1,5 \%$ par an pour les oléoprotéagineux (hypothèse d'extensification).

- après exclusion des petits producteurs non soumis à l'obligation du gel de $15 \%$ des surfaces, la proportion de terres gelées s'établit à $9,8 \%$ de la surface totale en céréales et oléoprotéagineux. Le coefficient de passage surface-quantité, traduisant la tendance des producteurs à geler les terres les moins fertiles, est fixé à 0,90 .

Sous ces hypothèses et en tenant compte des diverses interactions, on obtient les variations des prix des matières premières utilisées dans l'alimentation animale (tableau 1).

Par ailleurs, il faut avoir à l'esprit que la baisse du coût de l'alimentation animale devrait se traduire par un effet d'expansion de la production communautaire de porcs, volailles et œufs. D'après les simulations effectuées à l'aide de MISS, cette dernière devrait augmenter de $8,5 \%$ entre $1992 / 93$ et 1995/96.

La simulation des effets de la réforme de la PAC sur la hiérarchie des prix des matières premières entrant dans la ration alimentaire aboutit ainsi à une forte baisse du prix des 
Tableau 1. Variations (en \%) prévisibles des prix des principales matières premières de 1993/94 à 1995/96 (Source : Guyomard et Mahé 1993, Guyomard et al 1993).

\begin{tabular}{|c|c|c|c|}
\hline \multicolumn{2}{|c|}{ Céréales } & $\begin{array}{c}\text { Produits } \\
\text { de substitution }\end{array}$ & Tourteaux \\
\hline Blé & -39 & Corn gluten feed -23 & \\
\hline $\begin{array}{l}\text { Maïs } \\
\text { Orges }\end{array}$ & $\begin{array}{l}-34-34 \\
-30\end{array}$ & $\begin{array}{ll}\text { Manioc }^{(1)} & -32 \\
\text { Autres } & -27\end{array}$ & -9 \\
\hline
\end{tabular}

(1) Pour le manioc on fait l'hypothèse d'une suppression de l'accord d'autolimitation et d'un maintien du droit de douane actuel $(6 \%)$.

céréales, une diminution un peu plus faible de celui des produits de substitution des céréales (PSC) et une réduction limitée du prix du soja.

\section{2 / Hypothèses de formulation des régimes}

\section{1 / Choix des matières premières}

Le nombre de matières premières pouvant entrer dans la formulation des aliments pour le porc est très important. De plus, il est variable en fonction des régions de production et de la taille de l'entreprise. Aussi nous avons choisi de sélectionner 20 matières premières représentatives de celles couramment utilisées pour le porc à l'engraissement (tableau 2) et représentant, à elles seules, près de $80 \%$ de celles utilisées en pratique en France. Les caractéristiques nutritionnelles retenues pour les simulations sont celles des tables INRA (1989). La teneur en énergie nette (EN) est déterminée à l'aide des équations proposées par Noblet et al (1989) et les teneurs en acides aminés digestibles sont calculées à l'aide des coefficients de digestibilité vraie (Rhône Poulenc Nutrition Guide, 1989).

\section{2 / Prix des matières premières}

Les effets des variations de prix en termes à la fois de composition de la ration et de coût de l'aliment ont été estimés en envisageant la période présente et la situation une fois la réforme de la PAC achevée. A chaque fois, deux variantes ont été considérées. Les prix des matières premières correspondant à chacune de ces variantes sont présentés dans le tableau 2.

\section{La période récente}

Les deux variantes étudiées au cours de la période récente ont trait au $2^{\text {e }}$ trimestre et au $3^{\text {e }}$ trimestre 1993 sur la base des cours constatés.

Le $2^{\text {e }}$ trimestre 1993 est caractéristique d'une situation où les effets de la réforme de la PAC ne sont pas encore sensibles. Le rapport entre le prix des céréales et le prix du soja correspond à peu près à la référence de base avant réforme. Ceci tient au fait que les mesures de gel de terre avec les primes d'accompagnement et les nouveaux prix des pro-

Tableau 2. Prix des matières premières (FF/quintal) dans les différents contextes de prix.

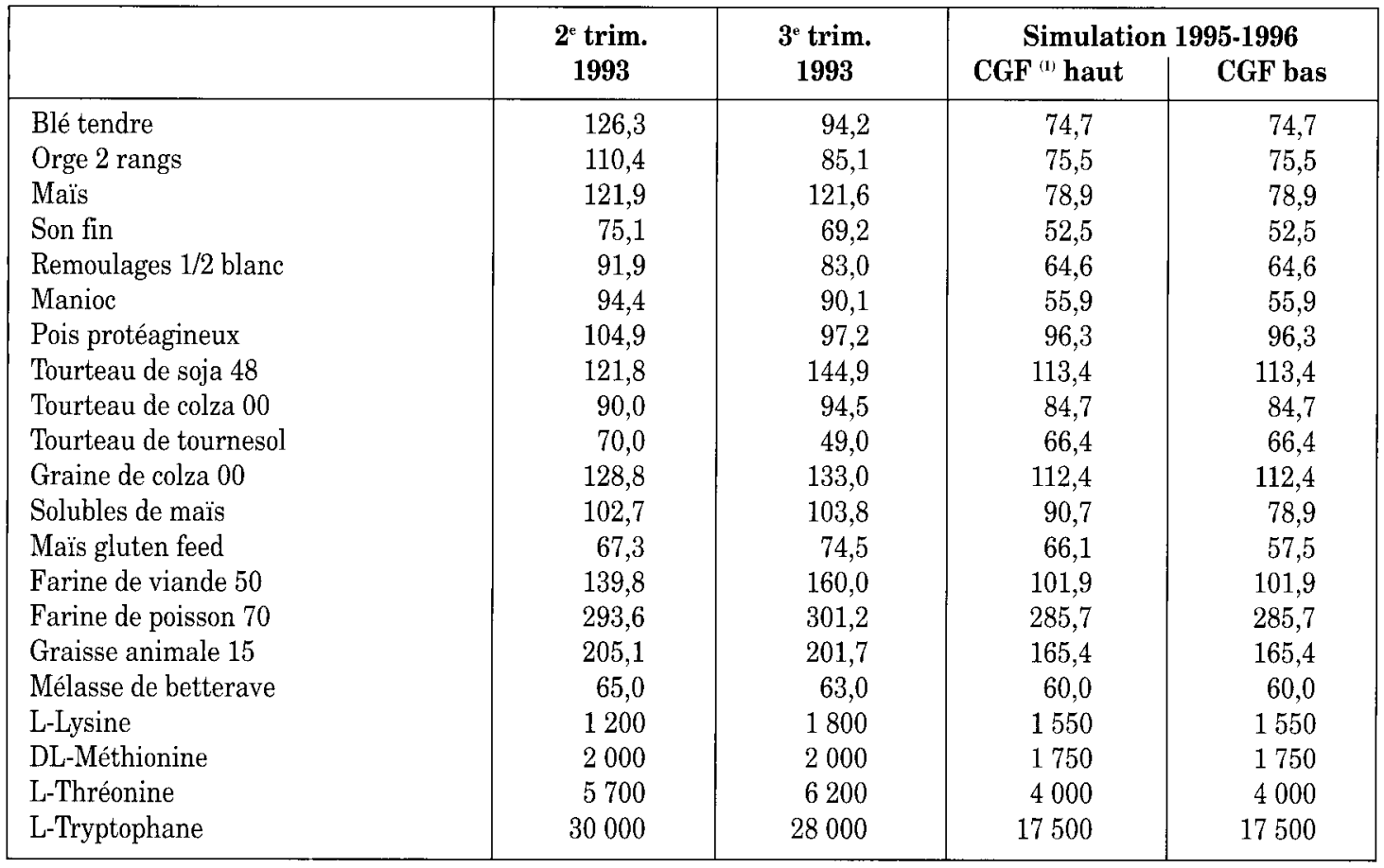

(1) CGF Corn Gluten Feed, voir dans le texte. 
duits agricoles ne sont entrés en vigueur qu'au $1^{\text {er juillet } 1993 .}$

Le $3^{e}$ trimestre 1993 est donc marqué par la baisse du prix des céréales. Mais, par ailleurs, les perturbations météorologiques intervenues aux Etats-Unis, avec de fortes inondations, se sont traduites par une baisse de l'offre de soja et, par conséquent, une hausse des cours. Il y a une conjonction assez particulière entre l'évolution du prix des tourteaux de soja et des céréales, favorisant un recours assez fort à ces dernières. La figure 1 , relative au rapport des prix entre céréales et soja, visualise clairement ces deux situations.

\section{La saison 1995-1996}

Dans ce cas, on applique aux prix d'achat des matières premières, observés sur les marchés français en 1992/93, les baisse de prix simulées à l'aide de MISS. Les deux variantes envisagées ne diffèrent que par la variation du prix du corn gluten feed à l'échéance de 95/96. En effet, le caractère de co-produit du CGF rend son coût très faible et fait que son offre est très inélastique puisqu'elle dépend plus des prix des produits finis que du prix du co-produit lui-même. Dans ces conditions, une baisse de la demande communautaire de CGF entraîne un fort ajustement à la baisse de son prix $(-23 \%$, tableau 1). Pour la catégorie autres PSC (autres que CGF et manioc), la baisse est de $27 \%$.

Cette baisse du prix du CGF de $23 \%$ correspond à la $1^{\text {re }}$ variante. La seconde prend en compte le contexte américain. En effet, les prix relativement intéressants obtenus par les producteurs américains de CGF sur le marché européen avaient gommé la demande intérieure des Etats-Unis. La forte baisse du prix du CGF à l'issue de la réforme de la PAC, telle qu'elle apparaît dans les simulations de MISS, pourrait modifier cet état de fait. Avec un faible prix, le CGF pourrait devenir attractif pour les utilisateurs américains dont la demande pourrait augmenter.
De ce fait, la baisse du prix de ce produit pourrait être freinée. Dans cette hypothèse, la baisse est fixée à $11 \%$.

\section{3 / Contraintes nutritionnelles et formulation des régimes}

Nous avons retenu trois hypothèses de conduite alimentaire en engraissement, correspondant aux différentes situations que l'on peut rencontrer en élevage.

- Distribution d'un aliment "unique", entre 25 et $105 \mathrm{~kg}$ de poids vif, permettant de couvrir les besoins en acides aminés tout au long de la période de croissance. Les besoins des animaux ayant tendance à diminuer au cours de la période d'engraissement, cette conduite s'accompagne d'un apport excessif de protéines en fin d'engraissement. C'est la conduite la plus fréquente dans les élevages.

- Adaptation de l'aliment à chacune des périodes de croissance (25-60 kg de poids vif) et de finition $(60-105 \mathrm{~kg}$ de poids vif). Cette conduite permet une meilleure adaptation de l'apport de protéines aux besoins des animaux.

- Alimentation "multiphase" basée sur le mélange progressif de deux aliments, correspondant respectivement aux besoins au début $(25 \mathrm{~kg}$ de poids vif) et à la fin (105 kg de poids vif) de la période d'engraissement. Cette conduite permet l'ajustement quasi parfait de l'apport protéique aux besoins des animaux. C'est une conduite rencontrée dans certains élevages disposant de systèmes automatiques d'alimentation.

Les contraintes nutritionnelles correspondant à chacune de ces trois conduites alimentaires sont rapportées au tableau 3. Elles sont basées sur les recommandations INRA (1989). Les besoins en acides aminés sont exprimés en acides aminés digestibles et les besoins énergétiques en énergie nette. Les limites maximales d'incorporation des différentes matières premières sont rapportées au tableau 4. Une limite globale maximale de

Figure 1. Evolution du rapport de prix entre les céréales et le tourteau de soja.

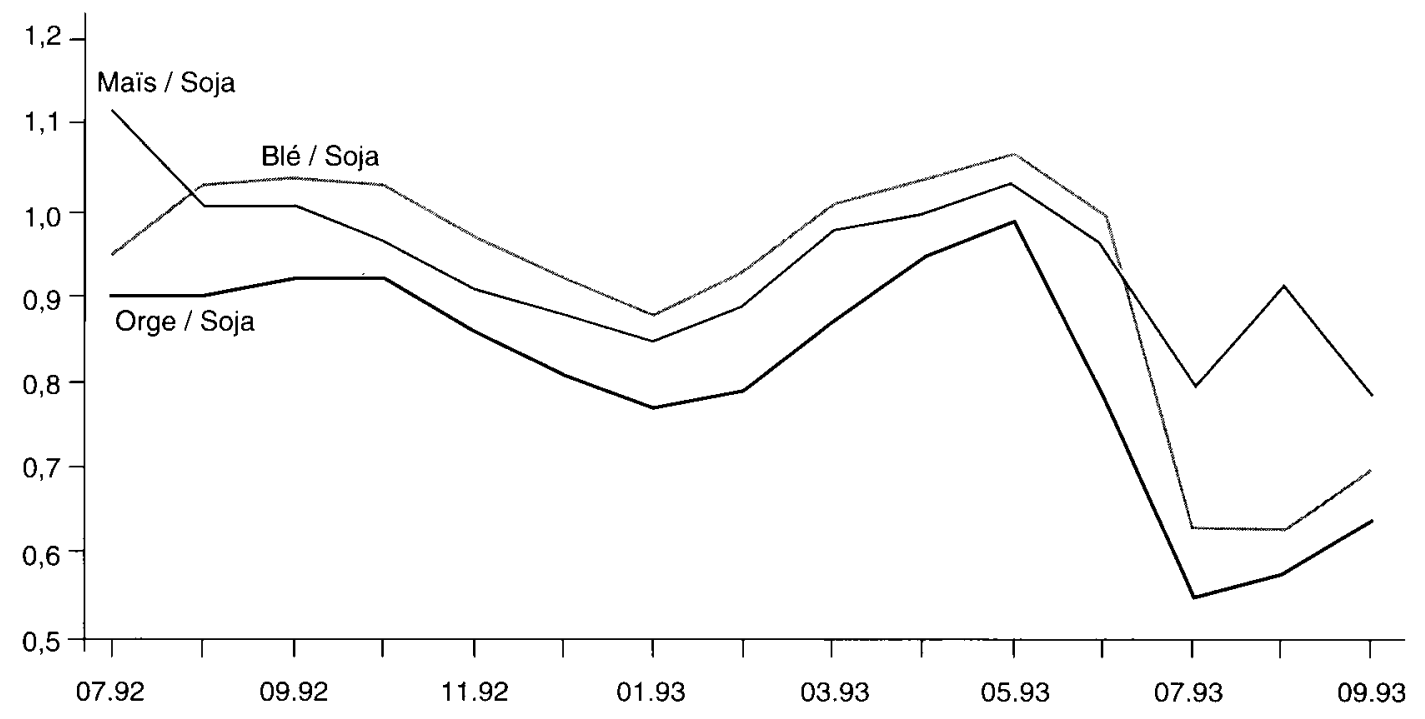


Tableau 3. Contraintes nutritionnelles "retenues pour la formulation des régimes.

\begin{tabular}{|l|c|r|r|r|r|r|r|r|}
\hline & \multicolumn{2}{|c|}{ Unique } & \multicolumn{2}{c|}{ Croissance } & \multicolumn{2}{c|}{ Finition } & \multicolumn{2}{c|}{$\begin{array}{c}\text { Finition } \\
\text { multiphase " } \\
\text { Min }\end{array}$} \\
& Min & Max & Min & Max & Min & Max & \multicolumn{2}{c|}{ Max } \\
\hline Energie nette (kcal/kg) & 2250 & & 250 & & 2250 & & 250 & \\
MAT (\%) & 13,0 & 20,0 & 13,0 & 20,0 & 12,0 & 20,0 & 12,0 & 20,0 \\
Matières grasses (\%) & & 6,0 & & 6,0 & & 6,0 & & 6,0 \\
Acide linoléique (\%) & & 1,5 & & 1,6 & & 1,2 & & 1,2 \\
Cellulose brute (\%) & 2,0 & 5,0 & 2,0 & 5,0 & 2,0 & 5,0 & 2,0 & 5,0 \\
Matières minérales (\%) & & 7,5 & & 7,5 & & 7,5 & & 7,5 \\
Calcium (\%) & 0,85 & & 0,90 & & 0,80 & & 0,80 & \\
Phosphore total (\%) & 0,60 & & 0,65 & & 0,50 & & 0,50 & \\
AA digestibles (\%) & & & & & & & & \\
- Lysine & 0,765 & & 0,790 & & 0,672 & & 0,600 & \\
- Méthionine & 0,229 & & 0,237 & & 0,202 & & 0,172 & \\
- AA soufrés & 0,459 & & 0,474 & & 0,403 & & 0,343 & \\
- Thréonine & 0,489 & & 0,505 & & 0,430 & & 0,365 \\
- Tryptophane & 0,145 & & 0,150 & & 0,128 & & 0,109 & \\
\hline
\end{tabular}

(1) Min : teneur minimale dans le régime; Max : teneur maximale dans le régime.

$25 \%$ est également fixée sur les «issues de céréales " tout comme pour les matières premières riches en cellulose brute dont la somme ne doit pas dépasser $15 \%$. Les normes maximales d'incorporation diffèrent entre les systèmes d'alimentation dans la mesure où les animaux les plus âgés sont généralement moins sensibles à l'incorporation de matières premières " à risque ".

A partir des prix et des caractéristiques de chacune des matières premières, on calcule ensuite, par programmation linéaire, la formule alimentaire la moins coûteuse, en tenant compte des contraintes d'incorporation et des besoins des animaux. Les formulations au moindre coût ( $\mathrm{FF}$ /quintal) ont été réalisées à l'aide du logiciel " PORFAL 》 (ITP, INRA, 1992). Pour chaque contexte de prix des matières premières, on détermine la formule au coût minimal, sans contrainte sur la teneur en protéines. On fait ensuite varier la contrainte de teneur en protéines de $12 \%$ à $19 \%$ par pas de 0,5 point, de façon à détermi- ner la composition et le prix de l'aliment en fonction de sa teneur en protéines.

\section{4 / Détermination de la relation entre le coût alimentaire et le rejet azoté}

Afin de prendre en compte l'influence des performances de production sur le coût alimentaire et les rejets azotés, trois hypothèses d'indice de consommation (IC) ont été retenues : un IC de $3,0 \mathrm{~kg}$ d'aliment/kg de gain de poids (moyenne des élevages) et des IC de 2,8 et 3,2 correspondant respectivement aux performances du tiers supérieur et du tiers inférieur des élevages français. Le coût alimentaire par porc produit est obtenu en multipliant le prix unitaire de l'aliment par l'IC et par le gain de poids vif des animaux $(25-105 \mathrm{~kg}$ de poids vif). Il s'agit donc d'un coût matières premières ne prenant pas en compte les charges de fabrication et de transport des aliments. Pour les stratégies d'ali-

Tableau 4. Contraintes maximales d'incorporation de certaines matières premières ou de groupes de matières premières (\%).

\begin{tabular}{|c|c|c|c|c|c|c|c|c|c|}
\hline & \multicolumn{3}{|c|}{ Unique } & \multicolumn{3}{|c|}{ Croissance } & \multicolumn{3}{|c|}{ Finition } \\
\hline Blé tendre & 40 & & & 40 & & & 40 & & \\
\hline Maïs & 40 & & & 40 & & & 40 & & \\
\hline Orge & 40 & & & 40 & & & 40 & & \\
\hline Pois protéagineux & 25 & & & 25 & & & 25 & & \\
\hline Tourteau de colza 00 & 12 & & & 10 & & & 14 & & \\
\hline Tourteau de tournesol & 5 & 15 & & 5 & 15 & & 5 & 15 & \\
\hline Son de blé & 15 & & & 10 & & & 15 & & \\
\hline Remoulage & 25 & & & 25 & & & 25 & & \\
\hline Soluble de maïs & 10 & & 25 & 10 & & 25 & 10 & & 25 \\
\hline Maïs gluten feed & 15 & & & 10 & & & 15 & & \\
\hline Manioc & 25 & & & 25 & & & 30 & & \\
\hline Graines de colza & 3 & & & 3 & & & 4 & & \\
\hline Mélasse de betterave & 7 & & & 6 & & & 8 & & \\
\hline Farine de viande & 4 & & & 4 & & & 5 & & \\
\hline Graisses animales & 4 & & & 4 & & & 4 & & \\
\hline
\end{tabular}


mentation basées sur deux aliments, le prix de l'aliment est déterminé en tenant compte des quantités consommées à chaque période, à savoir 1/3 en croissance et $2 / 3$ en finition.

L'excrétion azotée est calculée par différence entre l'apport azoté alimentaire et la rétention azotée corporelle selon le modèle proposé par Dourmad et al (1992). La quantité d'azote présente dans le lisier est ensuite déterminée en tenant compte des émanations gazeuses, qui s'élèvent à environ $22 \%$ de l'azote excrété dans le cas d'un bâtiment sur caillebotis intégral (Guillou et al 1993).

\section{3 / Analyse des résultats}

\section{1 / Situation au prix minimum}

Le tableau 5 récapitule les résultats obtenus dans le cas de la formulation au moindre coût, sans contrainte sur la teneur en protéines du régime. Ceci permet d'évaluer, en fonction du contexte économique, le coût alimentaire par porc et le niveau d'excrétion azotée pour les différentes situations de performances et de conduite alimentaire.

\section{Influence de la conduite alimentaire}

Quel que soit le contexte, l'alimentation "multiphase" permet d'obtenir le coût alimentaire le plus bas. Ce dernier est plus élevé pour l'aliment " unique " et intermédiaire pour l'alimentation " croissance-finition ". Ainsi, dans le contexte du $2^{2}$ trimestre 93, l'écart de coût alimentaire entre la conduite «multiphase » et l'aliment « unique " est d'environ $5 \mathrm{~F}$ /porc. Lorsque le prix des sources de protéines est élevé (contexte du $3^{\mathrm{e}}$ trimestre 93 ) cet écart augmente et passe à près de $10 \mathrm{~F} /$ porc.

La modification de la conduite alimentaire se répercute également sur la composition de l'aliment à l'optimum économique. Le passage à une alimentation "multiphase » entraî- ne une réduction de la teneur moyenne en protéines, la situation "croissance-finition " se trouvant en situation intermédiaire. Les rejets azotés étant directement liés à la teneur en protéines de l'aliment, ceci s'accompagne d'une réduction de l'excrétion azotée de 200 à $500 \mathrm{~g} /$ porc, la situation la plus favorable étant obtenue dans le cas de l'alimentation « multiphase».

\section{Influence du contexte économique}

En terme de coût alimentaire, la ration la moins onéreuse est obtenue, quel que soit le type d'alimentation, à l'horizon 95/96 avec une hypothèse de forte baisse du CGF. Par rapport à la situation représentative de la période avant la réforme de la PAC ( $2^{\mathrm{e}}$ trimestre 1993), le gain induit par la nouvelle hiérarchie des prix s'établit entre 50 et $60 \mathrm{~F}$ par porc, soit une réduction de près de $25 \%$.

En terme de rejets azotés les formules les moins polluantes apparaissent lorsque les sources de protéines sont désavantagées par rapport aux céréales : tel est le cas au $3^{\text {e }}$ trimestre 1993 avec des cours élevés du soja, ou à l'horizon $95 / 96$, avec une hypothèse de faible baisse du prix du CGF. Cependant, cette réduction reste très conditionnelle à l'incorporation de produits de substitution. Elle est de $9 \%$ dans l'hypothèse d'une baisse modérée du prix du CGF et seulement de $3 \%$ si la baisse est importante. Ceci est illustré par la figure 2 qui met en relation le coût alimentaire et l'excrétion azotée dans les 4 contextes économiques. La nouvelle PAC semble donc intervenir favorablement sur les rejets azotés, mais il est difficile de quantifier cet effet dans la mesure où il est très dépendant de l'évolution du marché des produits de substitution aux céréales.

\section{Influence des performances techniques}

Il est clair que l'on a toujours intérêt à diminuer l'indice de consommation puisque

Tableau 5. Niveau des rejets d'azote ( $\mathrm{kg} \mathrm{N}$ dans l'effluent entre 25 et $105 \mathrm{~kg}$ de poids vif) et coût de l'alimentation par porc (F) en fonction du contexte de prix, de la conduite alimentaire et de l'indice de consommation (IC).

\begin{tabular}{|c|c|c|c|c|c|c|c|c|}
\hline & \multicolumn{4}{|c|}{1993} & \multicolumn{4}{|c|}{$1995 / 1996$} \\
\hline & \multicolumn{2}{|c|}{$2^{\circ}$ trismestre } & \multicolumn{2}{|c|}{$3^{e}$ trimestre } & \multicolumn{2}{|c|}{ forte baisse CGF } & \multicolumn{2}{|c|}{ faible baisse CGF } \\
\hline & $\mathbf{N}$ & Coût & $\mathbf{N}$ & Coût & $\mathbf{N}$ & Coût & $\mathbf{N}$ & Coût \\
\hline Aliment unique & 348 & 25 & 326 & 317 & 34 & 170 & 340 & 173 \\
\hline IC $: 3,0$ & 3,83 & 242 & $\begin{array}{l}3,20 \\
3,60\end{array}$ & 233 & $\begin{array}{l}3,41 \\
3,83\end{array}$ & $\begin{array}{l}170 \\
183\end{array}$ & $\begin{array}{l}0,40 \\
3,75\end{array}$ & $\begin{array}{l}180 \\
185\end{array}$ \\
\hline IC $: 3,2$ & 4,18 & 257 & 3,94 & 248 & 4,18 & 195 & 4,10 & 198 \\
\hline Croiss-finition & & & & & & & & \\
\hline $\mathrm{IC}: 2,8$ & 3,19 & 223 & 3,00 & 214 & 3,28 & 167 & 3,10 & 170 \\
\hline IC $: 3,0$ & 3,53 & 239 & 3,33 & 229 & 3,63 & 179 & 3,43 & 182 \\
\hline IC $: 3,2$ & 3,83 & 255 & 3,65 & 244 & 3,97 & 191 & 3,76 & 194 \\
\hline Multiphase & & & & & & & & \\
\hline IC $: 2,8$ & 3,00 & 221 & 2,82 & 209 & 3,01 & 165 & 2,82 & 167 \\
\hline IC $: 3,0$ & 3,33 & 237 & 3,13 & 224 & 3,33 & 176 & 3,13 & 179 \\
\hline IC : 3,2 & 3,65 & 252 & 3,44 & 239 & 3,65 & 188 & 3,44 & 191 \\
\hline
\end{tabular}


cela permet à la fois de gagner sur le coût de l'alimentation et de diminuer les rejets. Ainsi, à l'horizon 95/96, quelle que soit l'hypothèse de variation du prix du CGF, une réduction de l'indice de consommation de 3,0 à 2,8 permet un gain de 11 à $13 \mathrm{~F}$ par porc $(-7 \%)$ et diminue les rejets de 0,31 à $0,36 \mathrm{~kg}(-10 \%)$.

\section{2 / Incidence d'une réduction du taux de protéines}

L'amélioration de la stratégie d'alimentation (« multiphase» ou « croissance-finition» versus "alimentation unique ") permet donc de réduire le rejet azoté tout en diminuant le coût alimentaire. La seconde voie de réduction du rejet azoté consiste à améliorer l'équilibre du régime en acides aminés, de façon à pouvoir réduire sa teneur en protéines et donc, in fine, le rejet azoté. En formulation, ceci nécessite d'introduire une contrainte supplémentaire sur la teneur maximale en protéines; c'est le coût de cette contrainte que nous allons maintenant étudier. Les résultats des simulations sont rapportés sous forme graphique dans les figures 2 et 3.

Il apparaît clairement sur ces graphiques, comme on l'a vu précédemment, que la solution optimale en termes de coût alimentaire est influencée par le contexte de prix (figure 2) et la stratégie d'alimentation (figure 3 ). Lorsque l'on s'éloigne de cette solution optimale, en particulier lorsque l'on cherche à réduire la teneur en protéines de façon à limiter le rejet azoté, le coût alimentaire augmente. Cependant l'ampleur de cette augmentation varie suivant les situations.

Tout d'abord, en fonction de la stratégie d'alimentation, on constate une modification de la stabilité de la solution optimale (figure 3). Ainsi, avec l'alimentation « croissancefinition " et de façon encore plus marquée avec une alimentation "multiphase ", il existe une zone relativement large dans laquelle le coût alimentaire est peu influencé par la teneur en protéines. Des variations du rejet azoté sont donc possibles, sans modification du coût alimentaire. En revanche, avec un aliment "unique », l'optimum est plus marqué et toute réduction de la teneur en protéines entraîne une augmentation du coût.

Le contexte de prix des matières premières influence également la sensibilité du coût alimentaire à la teneur en protéines (figure 2). Il semble en effet que dans une situation de prix élevés des sources de protéines ( $3^{\mathrm{e}}$ trimestre 1993), l'optimum soit plus marqué. Ceci se conçoit facilement dans la mesure où dans ce contexte la teneur en protéines à l'optimum est plus faible et toute réduction supplémentaire devient alors plus coûteuse.

A partir de ces différents éléments on peut quantifier pour chaque conduite alimentaire le coût supplémentaire, par rapport à l'optimum, d'une réduction de 500 ou $1000 \mathrm{~g} /$ porc du rejet d'azote dans le lisier (tableau 6). Cela correspond à une réduction de l'excrétion totale (lisier + ammoniac dans l'air), de 650
Figure 2. Influence du contexte économique sur le coût alimentaire et les rejets azotés, en alimentation de type "multiphase».

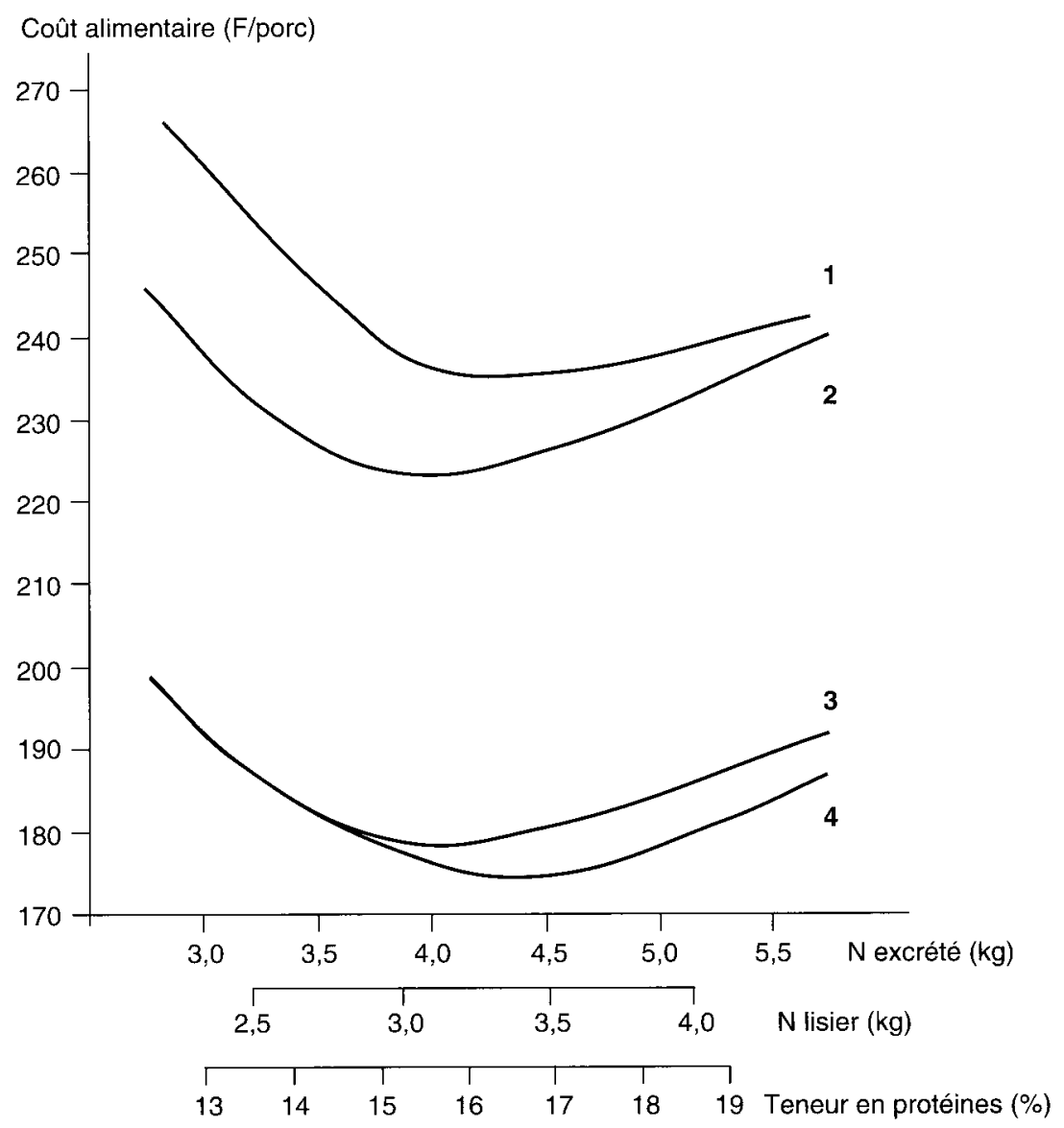

Tableau 6. Coût à l'optimum et coût supplémentaire $(F)$ d'une réduction de 0,5 ou $1 \mathrm{~kg} /$ porc du rejet azoté dans le lisier ${ }^{(t)}$.

\begin{tabular}{|l|c|c|c|}
\hline & Unique & $\begin{array}{c}\text { Croissance } \\
\text { finition }\end{array}$ & Multiphase \\
\hline $2^{e}$ trimestre 93 & & & \\
coût à l'optimum & 245 & 239 & 237 \\
pour - 0,5 kg N & +9 & +2 & +0 \\
pour - 1,0 kg N & +26 & +18 & +8 \\
$\begin{array}{l}\text { 3e trimestre 93 } \\
\text { coût à l'optimum }\end{array}$ & 234 & 229 & 227 \\
pour - 0,5 kg N & +4 & +4 & +2 \\
pour - 1,0 kg N & +21 & +20 & +16 \\
95/96 faible & & & \\
baisse du CGF & & 182 & 180 \\
coût à l'optimum & 187 & +3 & +2 \\
pour - 0,5 kg N & +5 & +13 & +13 \\
pour - 1,0 kg N & +16 & & \\
$\begin{array}{l}\text { 95/96 forte } \\
\text { baisse du CGF }\end{array}$ & & 179 & 177 \\
coût à l'optimum & 184 & +2 & +0 \\
pour - 0,5 kg N & +8 & +12 & +9 \\
pour - 1,0 kg N & +19 & +12 \\
\hline
\end{tabular}

(1) Le rejet à l'optimum peut être différent en fonction du contexte et de la stratégie d'alimentation. 
Figure 3. Influence de la stratégie d'alimentation sur le coût alimentaire et les rejets azotés.

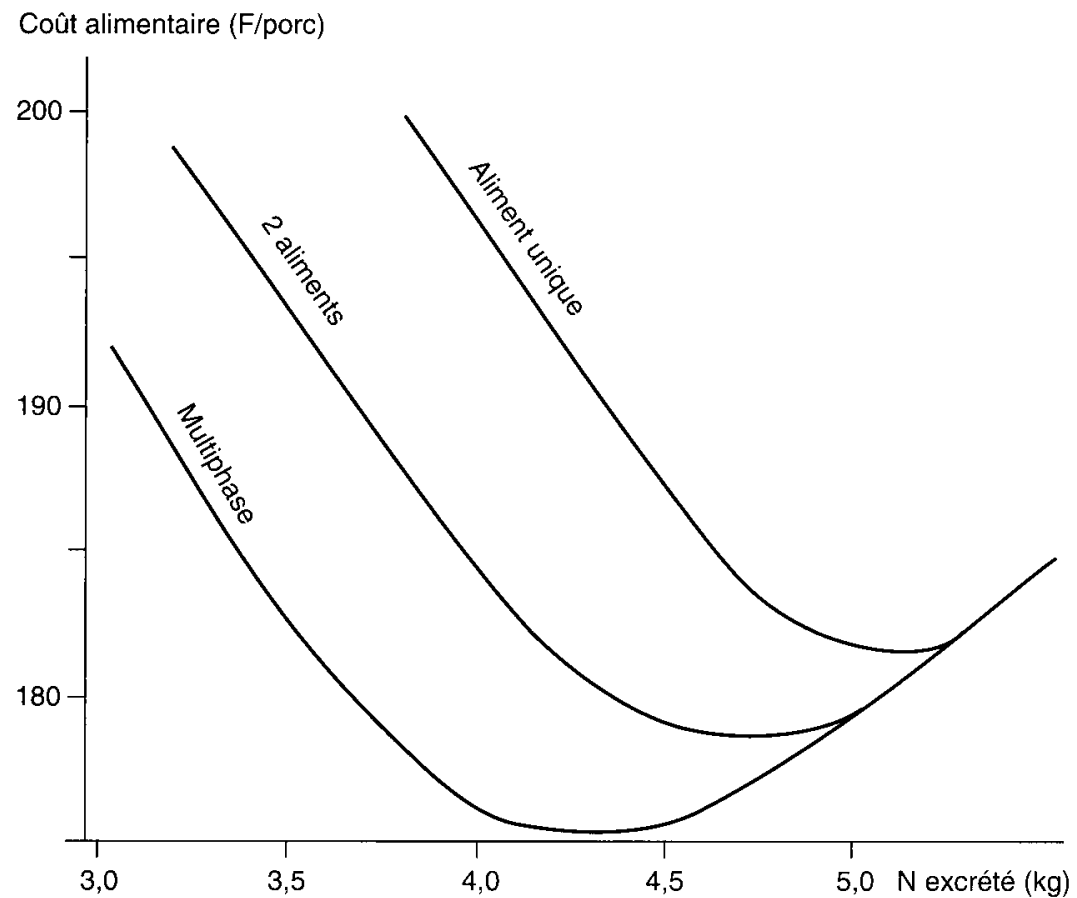

$$
2,5
$$

$$
3,0
$$

Teneur en protéines (\%)

ou 1300 g/porc. En moyenne, sur l'ensemble des contextes, l'augmentation de coût est de $4,10 \mathrm{~F} /$ porc pour une réduction de $500 \mathrm{~g} /$ porc d'azote dans le lisier et de $17 \mathrm{~F}$ pour une réduction de $1 \mathrm{~kg} /$ porc. Si l'on considère uniquement les stratégies basées sur deux aliments, les valeurs correspondantes sont de 1,9 et $13,6 \mathrm{~F} /$ porc. Lorsque l'on exprime ces valeurs en pourcentages, on peut calculer qu'une réduction de $14 \%$ des rejets azotés $(0,5 \mathrm{~kg})$ s'accompagne d'une augmentation d'environ $1 \%$ du coût alimentaire, alors que pour $28 \%$ de réduction des rejets $(1 \mathrm{~kg}) \mathrm{le}$ coût alimentaire augmente de $6 \%$.

\section{Conclusion}

En plus de ses effets sur le coût alimentaire, la réforme de la PAC induit des modifications importantes dans la formulation des régimes. Dès le $3^{e}$ trimestre 1993 , on constate une réduction de 1 à 1,5 point de la teneur en protéines des aliments, à l'optimum économique. Ceci s'accompagne d'une diminution de 0,2 à $0,4 \mathrm{~kg} /$ porc de l'excrétion azotée. A l'horizon $95 / 96$, on retrouve une situation relativement semblable dans l'hypothèse d'une baisse modérée du prix du CGF. Par contre, avec une baisse plus importante, de l'ampleur de celle prédite par le modèle MISS, il semblerait que la teneur en pro- téines à l'optimum tende à augmenter à nouveau pour atteindre une valeur intermédiaire entre celles des $2^{e}$ et $3^{\text {e }}$ trimestres 1993 . La teneur en protéines de l'aliment et l'importance du rejet azoté sont donc très sensibles aux rapports de prix entre sources de protéines et d'énergie. Ces variations peuvent être un frein à la mise en place par les industriels de gammes d'aliments à teneur limitée en protéines. En outre, il faut rappeler ici que, si la réforme de la PAC semble aller dans le sens d'une réduction de l'excrétion azotée par porc produit, elle pourrait également induire un accroissement de la production porcine, contribuant ainsi à l'augmentation du volume global d'excrétion azotée.

La modification de la stratégie d'alimentation permet de réduire le rejet azoté d'environ $500 \mathrm{~g} /$ porc, tout en réduisant le coût alimentaire de 8 à $13 \mathrm{~F} /$ porc ( 3 à $5 \%$ ). La modification de la stratégie d'alimentation est d'autant plus intéressante que les sources de protéines sont chères et que le potentiel de performance de l'élevage est élevé. Cependant, il faut rappeler que les conduites alimentaires mettant en œuvre plusieurs aliments («croissance-finition " ou «multiphases») induisent des investissements supplémentaires au niveau de l'élevage. En particulier, les possibilités de stockage doivent être augmentées et les systèmes de distribution adaptés de façon à permettre la distribution (et éventuellement le mélange) d'aliments différents en fonction du stade physiologique. Ceci nécessite également une meilleure maîtrise technique de la part de l'éleveur.

La seconde voie de réduction du rejet azoté, qui consiste à améliorer l'équilibre du régime en acides aminés, est techniquement très prometteuse. L'évaluation économique montre que cette approche s'accompagne d'une augmentation du coût alimentaire d'autant plus importante que l'on s'éloigne de la solution optimale. Une réduction du rejet azoté de $500 \mathrm{~g} /$ porc entraîne ainsi un coût supplémentaire d'environ $2 \mathrm{~F}$ /porc pour une stratégie basée sur 2 aliments contre plus de $4 \mathrm{~F} /$ porc pour un aliment unique.

Si l'on combine l'amélioration de la stratégie d'alimentation et l'amélioration de l'équilibre protéique du régime, on peut réduire le rejet azoté d'environ 20 à $25 \%$ (de 800 à $1000 \mathrm{~g} /$ porc) sans augmenter significativement le coût alimentaire. Restent cependant à considérer les amortissements liés à la modification du système d'alimentation.

Rappelons également que, indépendamment du contexte de prix des matières premières, l'amélioration de l'IC s'accompagne d'une réduction conjointe du coût alimentaire et du rejet azoté. Ainsi, lorsque l'IC passe de 3,2 à $2,8 \mathrm{~kg} / \mathrm{kg}$, le rejet azoté est réduit de $17 \%(0,65 \mathrm{~kg} /$ porc en moyenne $)$ et le coût alimentaire de $12 \%$ ( $26 \mathrm{~F} /$ porc en moyenne). Toutes les mesures ayant un effet favorable sur ce paramètre s'accompagneront donc d'une réduction des rejets azotés, en particulier l'amélioration du niveau génétique ou 
sanitaire de l'élevage ou encore des conditions de logement.

Sur le plan économique, diverses voies sont possibles pour faire coïncider optimum économique privé de l'éleveur et amélioration environnementale maximale.

On peut par exemple envisager de favoriser l'utilisation « d'aliments verts " à l'aide d'une subvention ou encore d'une réduction des taxes qui sont prélevées dans les exploitations en situation d'excédent. Cette approche est voisine de celle utilisée aux Pays-Bas à une certaine période.

On peut également considérer la possibilité d'une incitation à l'amélioration des systèmes de distribution de l'aliment. En effet, une fois ces systèmes disponibles sur l'élevage, l'optimum économique est obtenu pour un rejet moindre. Cette approche a de plus l'intérêt de rendre moins coûteuses les étapes ultérieures de réduction de la teneur en protéines de l'aliment.

Enfin, on peut penser que la seule prise en compte, dans le calcul du bilan azoté (donc des taxes éventuelles), des rejets effectifs des animaux et non de normes moyennes, comme actuellement, aurait un effet incitateur très important en faveur des approches préventives. On peut en évaluer l'intérêt à l'aide de l'exemple d'un élevage s'engageant dans cette voie en agissant à la fois sur les performances techniques et l'alimentation. On peut ainsi calculer que, pour un indice de consommation de $2,8 \mathrm{~kg} / \mathrm{kg}$, une teneur en muscle à l'abattage de $56 \%$ et une alimentation multiphase (teneur moyenne en protéines de $15 \%$ ), le rejet azoté dans le lisier (entre 30 et $106 \mathrm{~kg}$ de poids vif) est de seulement $2,2 \mathrm{~kg} /$ porc. Ceci correspond à une réduction par rapport à la norme du CORPEN ${ }^{11}$ de $1,3 \mathrm{~kg} /$ porc soit $37 \%$. On mesure bien, au travers de cet exemple, le pouvoir incitatif de cette approche.

(1) CORPEN : Comité d'Orientation pour la Réduction de la Pollution des Eaux par les Nitrates, les phosphates et les produits Phytosanitaires.

Les auteurs remercient la Commission des Communautés Européennes (DG VI, contrat CT92-0112) pour le soutien financier qu'elle a apporté à cette étude.

\section{Références bibliographiques}

Dourmad J.Y., Henry Y., 1994. Influence de l'alimentation et des performances sur les rejets azotés des pores. INRA Prod. Anim., 7 (4), 263-274.

Dourmad J.Y., Guillou D., Noblet J., 1992. Development of a calculation model for predicting the amount of $\mathrm{N}$ excreted by the pig : effect of feeding, physiological stage and performance. Livest. Prod. Sci., 31, 95-107.

Guillou D., Dourmad J.Y., Noblet J., 1993. Influence de l'alimentation, du stade physiologique et des performances sur les rejets azotés du porc à l'engrais, de la truie et du porcelet. Journées Rech. Porcine en France, 25, 307-314.

Guyomard H., Mahé L.P., 1993. La réforme de la PAC (projet de juillet 1991 et texte adopté en mai 1992) : Evaluation à l'aide du modèle MISS. Rapport pour la Commission des CE - DG VI. ENSA-INRA, Rennes, $47 \mathrm{pp}$.

Guyomard H., Le Mouël C., Surry Y., 1993. Les effets de la réforme de la PAC sur les marchés céréa- liers communautaires: Analyse exploratoire. Cahiers d'Economie et Sociologie Rurales, 17, 7-41.

INRA, 1989. L'alimentation des animaux monogastriques. Ed INRA, Paris.

ITP, INRA, 1992. Porfal. Manuel d'utilisation. Ed ITP, Paris.

Lenis N.P., 1987. Contribution of pig to N-pollution and possible solutions via feeding measures. In : Eurolysine/Gallenica feed industry symposium, Billund, Denmark, May 6th 1987, 8 pp.

Noblet J., Fortune H., Dubois S., Henry Y., 1989. Nouvelles bases d'estimation des teneurs en énergie digestible, métabolisable et nette des aliments pour le porc. INRA ed., Paris, 106 pp.

Rhône Poulenc Nutrition Guide, 1989. Formulation des aliments en acides aminés digestibles. Rhône Poulenc Animal Nutrition, Antony, 55 pp.
Reduction of nitrogen output from pig herds through better feeding : economical evaluation and effects of the new Common Agricultural Policy (CAP).

In regions where intensive animal production is found, the management and disposal of manure directly affects the extent and the risk of water pollution by nitrates and air pollution by ammonia. As an alternative or complement to the treat- ment of effluents, solutions have been sought to reduce nitrogen pollution at its source of production. One approach that has been pursued has been to better adapt protein available in feeds to animal needs. In the context of the reformed CAP, this paper presents an evaluation of the cost of such a preventive approach to nitrogen pollution control. The results show that the CAP reform has introduced important modifications in the 
formulation of feed rations. A 1 to 1.5 point reduction in the protein content of feeds is estimated to result in a diminution of nitrogen excretion by pigs in the range of between .2 to $.4 \mathrm{~kg} /$ pig. Further, it is shown that these results are very sensitive to the relationship between the prices of protein and energy. Modifications in the feeding strategy (number of diets) induce a decrease in nitrogen excretion in the range of $500 \mathrm{~g} / \mathrm{pig}$, as well as a reduction in the cost of the feedstuffs by 8 to $13 \mathrm{FF} / \mathrm{pig}(3-5 \%)$. However, this approach requires supplementary investments at the farm level for storage and distribution of the feeds. The improvement of the amino acid balance of the diet is generally associated with an increase in total feed costs. It is estimated that a reduction in nitrogen output by $500 \mathrm{~g} / \mathrm{pig}$ would involve additional costs of 2 to $4 \mathrm{FF} / \mathrm{pig}$. Jointly considering the benefits of improved feeding program and the improved protein balance, it is clear that nitrogen production can be reduced about 20 to $25 \%$, without substantilly increasing the cost of primary feed ingredients.

DOURMAD J.Y., LE MOUEL C., RAINELLI P., 1995. Réduction des rejets azotés des pores par la voie alimentaire : évaluation économique et influence des changements de la Politique Agricole Commune. INRA Prod. Anim., 8 (2), 135-144. 